\title{
LY294002 inhibits the malignant phenotype of osteosarcoma cells by modulating the phosphatidylinositol 3-kinase/Akt/fatty acid synthase signaling pathway in vitro
}

\author{
YANG ZHOU ${ }^{1 *}$, LIANG BO ZHU ${ }^{1 *}$, AI FEN PENG ${ }^{2}$, TAO FANG WANG ${ }^{1}$, XIN HUA LONG $^{1}$, \\ SONG GAO $^{1}$, RONG PING ZHOU ${ }^{3}$ and ZHI LI LIU ${ }^{1}$ \\ ${ }^{1}$ Department of Orthopedics, First Affiliated Hospital of Nanchang University, Nanchang, Jiangxi 330006; ${ }^{2}$ College of Humanities, \\ Jiangxi University of Traditional Chinese Medicine, Nanchang, Jiangxi 330006; ${ }^{3}$ Department of Orthopedics, \\ Second Affiliated Hospital of Nanchang University, Nanchang, Jiangxi 330006, P.R. China
}

Received December 18, 2013; Accepted September 24, 2014

DOI: $10.3892 / \mathrm{mmr} .2014 .2787$

\begin{abstract}
Increasing evidence suggests that fatty acid synthase (FASN) is crucial in the carcinogenesis of various types of tumor. In addition, the phosphatidylinositol 3-kinase (PI3K)/Akt signaling pathway, which is closely associated with cellular metabolism, affects cancer biology. However, whether the malignant phenotype of osteosarcoma (OS) cells is regulated by the PI3K/Akt/FASN signaling pathway and how the PI3K family specific inhibitor, 2-(4-morpholinyl)-8-phenyl-chromone (LY294002) affects the malignant phenotype of OS cells remains to be elucidated. In the present study, U2-OS and MG-63 cells were treated with LY294002 and subsequently western blot analysis was used to examine Akt, p-Akt and FASN protein expression. Additionally, FASN mRNA was detected by reverse transcription quantitative polymerase chain reaction. MTT and fluorescence-activated cell sorting assays were used to assess proliferation and apoptosis. Migration and invasion were investigated using wound healing and transwell invasion assays. The results demonstrated that LY294002 suppressed the PI3K/Akt/FASN signaling pathway. However, the malignant phenotypes of OS cells mentioned above were significantly inhibited. The present results indicated that LY294002 inhibits the malignant phenotype of OS cells via modulation of the $\mathrm{PI} 3 \mathrm{~K} / \mathrm{Akt} / \mathrm{FASN}$ signaling pathway in vitro and may be a new therapeutic strategy for the management of OS.
\end{abstract}

Correspondence to: Dr Zhi Li Liu, Department of Orthopedics, First Affiliated Hospital of Nanchang University, 17 Yong Wai Zheng Street, Nanchang, Jiangxi 330006, P.R. China

E-mail: zgm7977@163.com

*Contributed equally

Key words: 2-(4-morpholinyl)-8-phenyl-chromone, malignant phenotype, osteosarcoma, phosphatidylinositol 3-kinase/Akt/fatty acid synthase signaling pathway

\section{Introduction}

Osteosarcoma (OS) is one of the most common types of primary malignant bone tumor in childhood and adolescence (1). Despite the introduction of effective chemotherapy and improvements in surgical technology, which has increased the survival rate to approximately $65-75 \%$, pulmonary metastasis occurs in $40-50 \%$ of OS patients and remains the leading cause of mortality (2-3). Therefore, it is important to uncover the molecular mechanisms involved in OS progression, particularly pulmonary metastasis.

Mechanistically, OS development, as with other types of cancer, involves multiple genetic alterations, including oncogene activation and tumor-suppressor gene dysfunction. Over the previous several decades, substantial knowledge has been gained regarding the molecular alterations associated with OS carcinogenesis and several key signaling pathways, including fatty acid synthase (FASN)/human epidermal growth factor receptor 2/phosphatidylinositol 3-kinase (PI3K)/Akt (5), extracellular signal-regulated kinases-p38-JNK (6), nuclear factor- $\kappa \mathrm{B}(7)$ and the mitogen-activated protein kinase pathway (8) have been implicated in OS metastasis. Dysregulation of the genes associated with these pathways has been demonstrated to promote OS cell growth and metastasis.

PI3Ks are a family of related intracellular signal transducer enzymes, which have the capability to phosphorylate the hydroxyl group at position 3 of the inositol ring of phosphatidylinositol. PI3Ks are important in tumor cell differentiation, the cell cycle, apoptosis, growth and metastasis via the activation of Akt. Various studies have demonstrated that decreasing the activation of the PI3K/Akt signaling pathway inhibits cell growth and metastasis in various types of tumor $(9,10)$. Several small molecule inhibitors of the PI3K/Akt signaling pathway have been previously developed as promising antitumor treatments (11-14). Previous studies have demonstrated that inhibition of PI3K/Akt significantly suppresses OS cell growth, migration and invasion $(5,15,16)$. Therefore, we hypothesized that small molecule inhibitors of the PI3K/Akt signaling pathway may be novel alternative agents for the treatment of OS. 
The present study investigated the inhibition of the malignant phenotype of U2-OS and MG-63 cells by 2-(4-morpholinyl)-8-phenyl-chromone (LY294002), a commonly used pharmacological inhibitor that selectively inhibits the PI3K-Akt pathway, via downregulation of the $\mathrm{PI}$ KK/Akt/FASN signaling pathway.

\section{Materials and methods}

Cell lines and cell culture. The human OS cell lines, U2-OS and MG-63, were obtained from the American Type Culture Collection (Manassas, VA, USA) and cultured in Dulbecco's modified Eagle's medium (DMEM; HyClone, Waltham, MA, USA) supplemented with $10 \%$ fetal bovine serum (FBS; Sigma Aldrich, St. Louis, MO, USA) in a humidified atmosphere of $5 \% \mathrm{CO}_{2}$ at $37^{\circ} \mathrm{C}$.

Cell growth assay. MG-63 and U2-OS cell lines were cultured in 96-well tissue culture plates at a density of 5,000 cells/well in minimum essential medium (Solarbio, Beijing,China) containing 10\% FBS and 2 mM l-glutamine. Following attachment overnight, the medium was replaced and cells were incubated with increasing concentrations (5, 10, 20, 40, 80 and $160 \mu \mathrm{M}$ ) of LY294002 (Sigma Aldrich). Following treatment for $48 \mathrm{~h}$, MTT assays were performed at a wavelength of $490 \mathrm{~nm}$ in triplicate. The inhibition ratio was calculated and the concentration-viability curves were fitted by the OriginPro 7.5 program (OriginLab, Northampton, MA, USA). The half maximal inhibitory concentration $\left(\mathrm{IC}_{50}\right)$ values of LY294002 were determined. All experiments were performed in triplicate.

Fluorescence-activated cell sorting (FACS). MG-63 and $\mathrm{U} 2-\mathrm{OS}$ cells in the exponential growth phase were treated with varying concentrations of LY294002 for $24 \mathrm{~h}$. Cells were then fixed with $70 \%$ ethanol and stained with propidium iodide. FACS analysis was performed to determine the percentage of apoptotic cells and cell cycle distribution by using the EPICS XL flow cytometer (Beckman Coulter, Miami, FL, USA) and System II software V4.16 (Coulter Electronics, Miami, FL, USA). All experiments were performed in triplicate.

Invasion assay. The invasiveness of the OS cells was measured using BD BioCoat ${ }^{\mathrm{TM}}$ BD Matrigel ${ }^{\mathrm{TM}}$ Invasion Chambers (BD Bioscience, Franklin Lakes, NJ, USA) according to the manufacturer's instructions. The medium in the lower chamber contained $15 \%$ FBS as a source of chemoattractant. The cells were suspended in serum-free medium containing LY294002 (20 and $40 \mu \mathrm{M})$ and added to the upper chambers simultaneously $\left(2 \times 10^{3}\right.$ cells in $\left.0.1 \mathrm{ml}\right)$. The cells that passed through the Matrigel-coated membrane were stained with Diff-Quik (Sysmex, Kobe, Japan) and images were captured. Cell invasion was quantified by direct microscopic visualization and cell counting. Invading cells were counted from five randomly selected fields under an inverted microscope. The cells not treated with LY294002 were used as a control. Three independent experiments were performed.

Migration assay. Cell migration was assessed by determining the ability of the cells to move into a cellular space in a two-dimensional wound healing assay in vitro. In brief, the cells were cultured in six-well tissue culture plastic dishes at a density of $5 \times 10^{6}$ cells/well and then subsequently treated with LY294002 (20 and $40 \mu \mathrm{M}$ ) for $24 \mathrm{~h}$. The cells were denuded by dragging a rubber policeman (Fisher Scientific, Hampton, NH, USA) through the center of the plate well. The culture plates were rinsed with phosphate-buffered saline (PBS) and fresh quiescent medium alone or with $10 \%$ bovine serum albumin was added. The cells were subsequently incubated at $37^{\circ} \mathrm{C}$ for $24 \mathrm{~h}$. Images of the cells were captured at 0 and $24 \mathrm{~h}$ and the migrated distance was measured. The migration rate was counted from five randomly selected fields under an inverted microscope (Olympus, Tokyo, Japan). The cells not treated with LY294002 were used as a control. Three independent experiments were performed.

Western blot analysis. U2-OS and MG-63 cells in the exponential growth phase were treated with LY294002 $(20,40,80$ and $160 \mu \mathrm{M})$ for $24 \mathrm{~h}$. The cells were subsequently washed with cold PBS. Total cellular protein was extracted using radioimmunoprecipitation assay lysis buffer containing $60 \mu \mathrm{g} / \mathrm{ml}$ phenylmethanesulfonyl fluoride and the protein concentration was determined using a Bradford protein assay. Equal quantities of protein were electrophoresed by $10 \%$ SDS-PAGE (Solarbio) and transferred onto a pure nitrocellulose blotting membrane $(0.22 \mu \mathrm{m}$ pores; Solarbio). The membranes were blocked with $5 \%$ Difco skimmed milk for $1 \mathrm{~h}$ at room temperature and then incubated with primary antibodies (monoclonal rabbit anti-Akt, anti-p-Akt and anti-FASN IgG; 1:2,000; Santa Cruz Biotechnology, Inc., Santa Cruz, CA, USA) overnight at $4^{\circ} \mathrm{C}$. The membranes were washed prior to incubation with the appropriate peroxidase-conjugated secondary antibodies (monoclonal mouse anti-rabbit; 1:5,000; Santa Cruz Biotechnology, Inc.). The immune complexes were detected with a Pro-light HRP kit (Tiangen Biotech Co., Ltd., Beijing, China). All experiments were performed in triplicate.

Reverse transcription quantitative polymerase chain reaction (RT-qPCR). Semi-quantitative PCR was used to detect FASN mRNA levels. Total RNA was extracted from cells using TRIzol reagent (Invitrogen Life Technologies, Carlsbad, CA, USA). The total RNA concentration was determined by spectrophotometry at $260 \mathrm{~nm}$ and the purity was determined by calculating the $260 / 280$ ratio with a BioPhotometer (Eppendorf, Hamburg, Germany). The Two-Step kit (Promega Corporation, Madison, WI, USA) was used to obtain cDNA according to the manufacturer's instructions, which was subsequently used as the template for amplification. The following primers were used to amplify target sequences: FASN, forward 5'-AACTCCATGTTTGGTGTTTG-3' and reverse 5'-CACATGCGGTTTAATTGTG-3', 171 bp (Sangon Biotech Shanghai Co., Ltd., Shanghai, China); GAPDH, forward 5'-CAGGGCTGCTTTTAACTCTGGT-3' and reverse 5'-GATTTTGGAGGGATCTCGCT-3', 199 bp (Sangon Biotech Shanghai Co., Ltd.). Following amplification, DNA electrophoresis was performed on standard $1 \%$ agarose gels and DNA was labeled and visualized using ethidium bromide. Images were captured using the Canon Digital IXUS 900Ti 


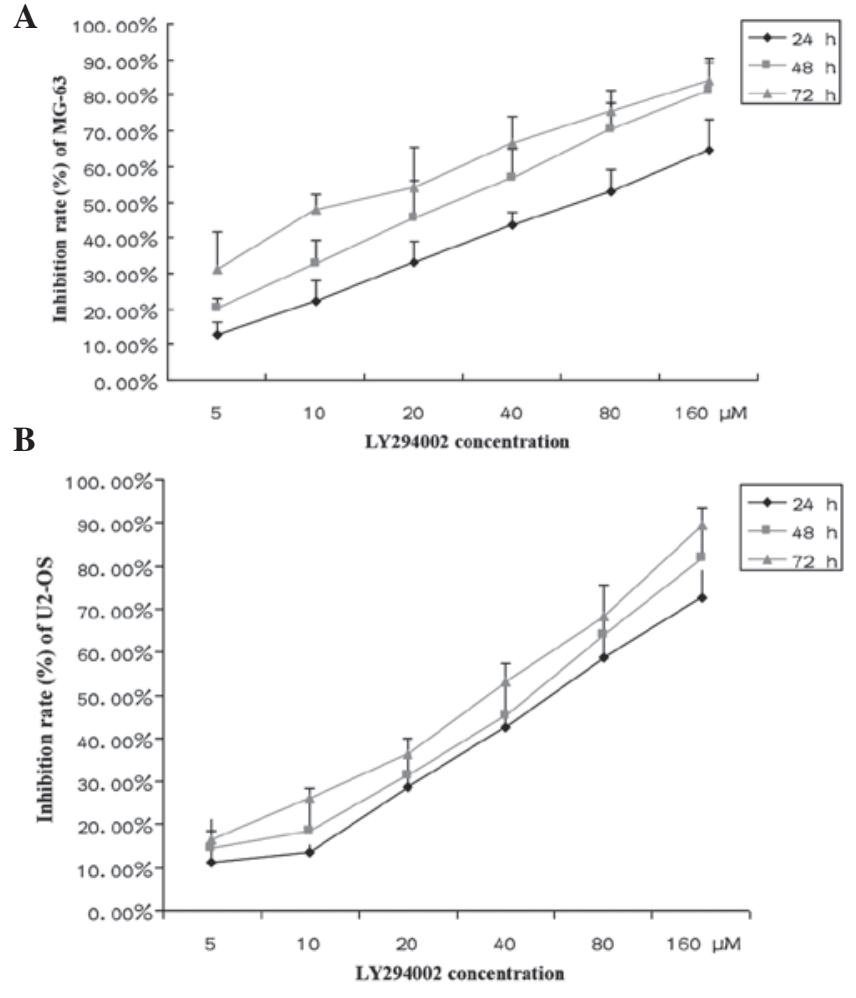

Figure 1. LY294002 suppresses MG-63 and U2-OS cell proliferation. (A and B) LY294002 inhibited MG-63 and U2-OS cell proliferation in a time- and dose-dependent manner and the $\mathrm{IC}_{50}$ values $(24 \mathrm{~h})$ were 62.98 and $55.2 \mu \mathrm{M}$, respectively.

(Canon Inc., Tokyo, Japan). All experiments were repeated six times.

\section{Results}

LY294002 inhibits OS cell growth. The inhibitory effect of LY294002 on the growth of U2-OS and MG-63 cell lines was examined using an MTT assay. The results revealed that MG-63 and U2-OS cell lines were sensitive to LY294002 and that LY294002 inhibits U2-OS and MG-63 cell proliferation in a time- and dose-dependent manner (Fig. 1). $\mathrm{IC}_{50}$ values at $24 \mathrm{~h}$ were 62.98 and $55.2 \mu \mathrm{M}$, respectively.

LY294002 induces OS cell apoptosis. FACS analysis was used to examine the mechanism of LY294002 inhibition of OS cell apoptosis. Gradient concentrations (20,40, 80 and $160 \mu \mathrm{M})$ of LY294002 were added to U2-OS and MG-63 cell cultures in the exponential growth phase. The treated and untreated cell samples were obtained and fixed for FACS analysis $24 \mathrm{~h}$ later. FACS analysis revealed that LY294002-induced apoptosis of U2-OS and MG-63 cells increased with increasing concentration of the inhibitor (Fig. 2). The data indicated that LY294002 induced OS apoptosis in a dose-dependent manner.

LY294002 inhibits OS cell invasion. Transwell invasion assays were used to examine the effect of LY294002 on U2-OS and MG-63 cell invasion. The results demonstrated that the proportion of invading cells of these cell samples treated with LY294002 was significantly lower than that of the untreated cells and the inhibitory effect of LY294002 on cell invasion
A
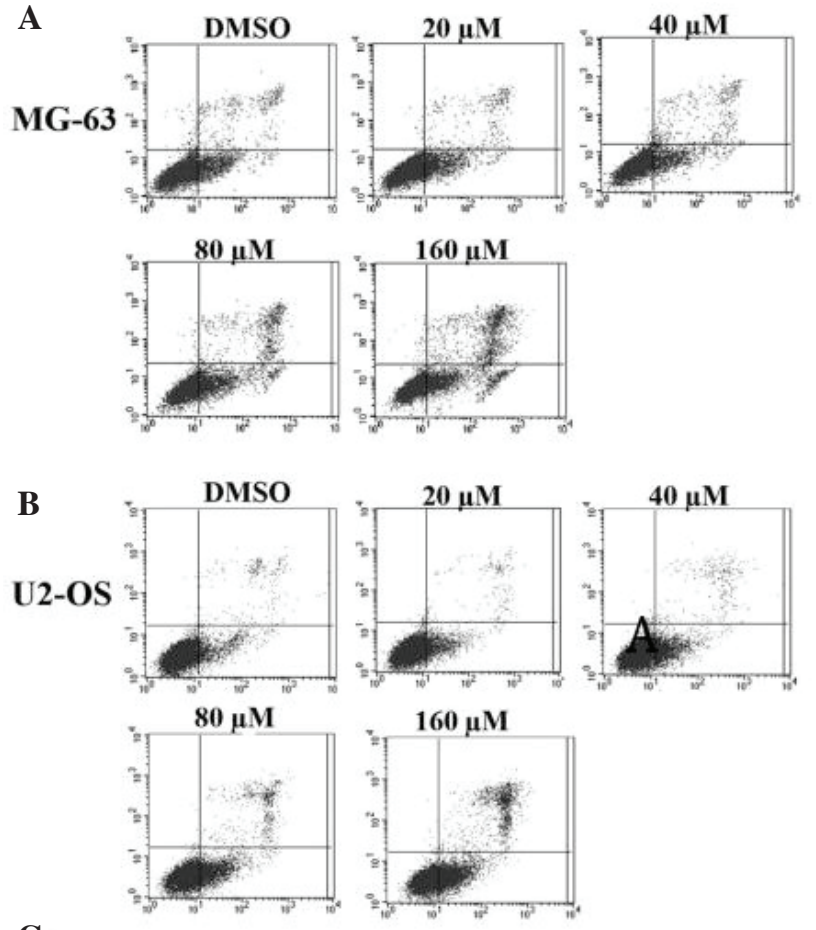

C

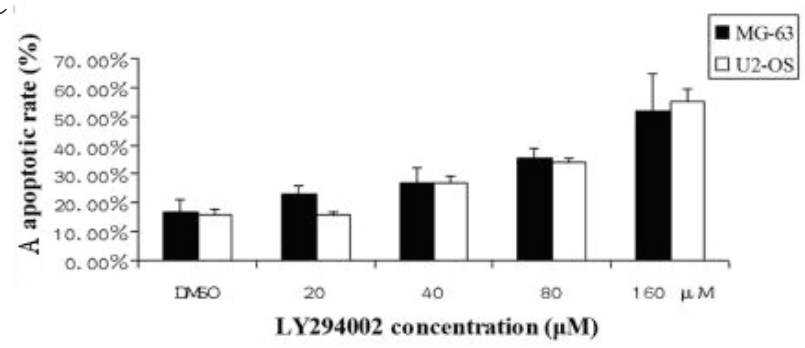

Figure 2. LY294002 induces U2-OS and MG-63 cell apoptosis. (A and B) LY294002 induced MG-63 and U2-OS cell apoptosis. (C) The histogram indicates that LY294002 accelerated osteosarcoma cell apoptosis in a dose-dependent manner. DMSO, dimethyl sulfoxide.

was increased with increasing concentration of the inhibitor (Fig. 3A and B). The data indicated that LY294002 inhibits OS cell invasion in vitro.

LY294002 decreases OS cell migration. The effect of LY294002 on the migration of U2-OS and MG-63 cells was evaluated using wound healing assays. The results revealed that the migration rate of cells treated with LY294002 was significantly lower when compared with that of the untreated cells and the migration rate decreased with increasing concentration of LY294002 (Fig. 3C and D). The results suggested that LY294002 inhibits OS cell migration.

LY294002 downregulates the activation of the PI3K/Akt/FASN pathway. To investigate the potential molecular mechanisms, RT-qPCR was performed to detect the expression of FASN mRNA in U2-OS and MG-63 cell lines to evaluate the inhibitory effect of LY294002 on FASN expression. The results revealed that the mRNA expression of FASN was significantly downregulated with increasing concentrations of LY294002 (Fig. 4A). Furthermore, the expression levels of p-Akt, Akt and FASN protein in U2-OS and MG-63 cell lines were measured using western blot analysis. The results revealed that $\mathrm{p}-\mathrm{Akt}$ 

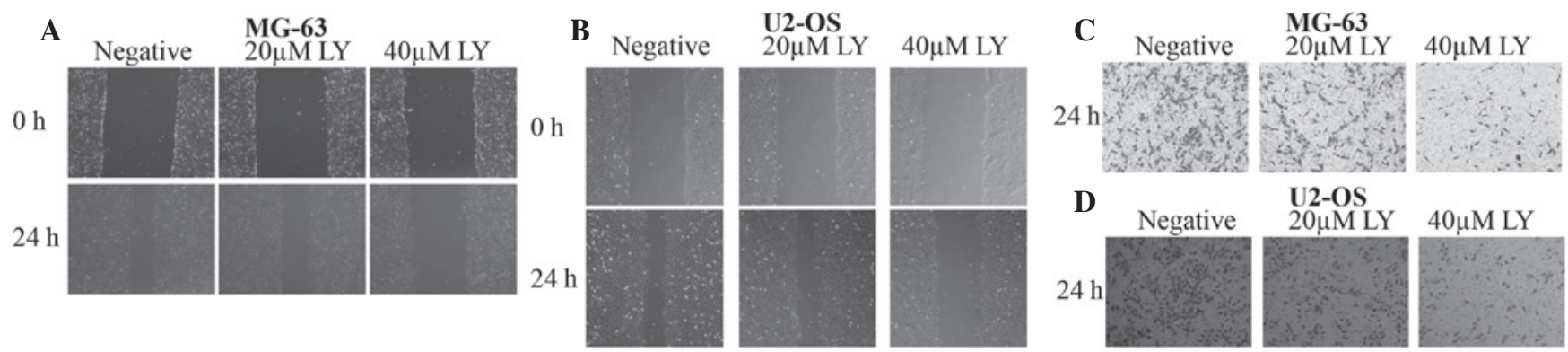

Figure 3. LY inhibits MG-63 and U2-OS cell migration and invasion. Representative images of the wound healing assays is shown for (A) MG-63 and (B) U2-OS cells. It indicates that LY suppressed MG-63 and U2-OS cell migration when compared with the negative group and the migrated cells decreased with increasing concentrations of LY (20 and $40 \mu \mathrm{M})$. Representative images of the invasion assays is shown for (C) MG-63 and (D) U2-OS cells. The results indicated that LY suppressed MG-63 and U2-OS cell invasion when compared with the negative group and the number of invasive cells decreased with increasing concentration of LY. LY, LY294002.

A

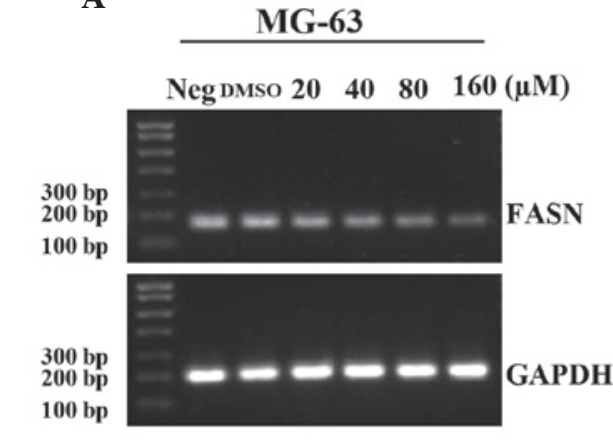

B

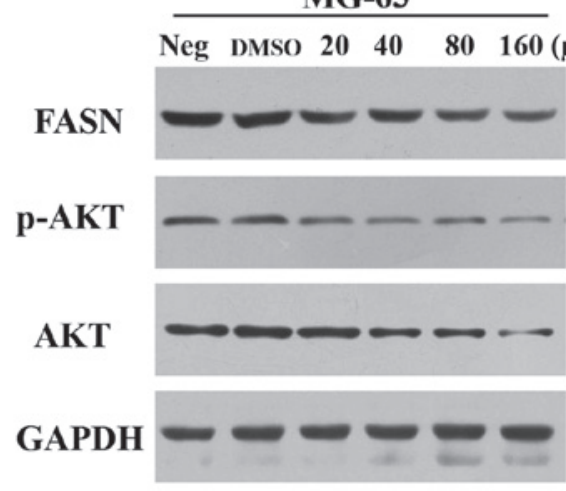

U2-OS

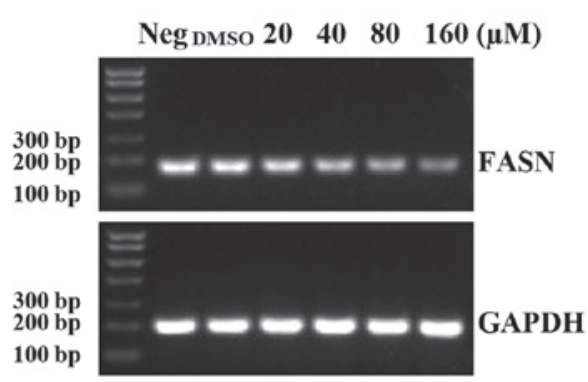

U2-OS

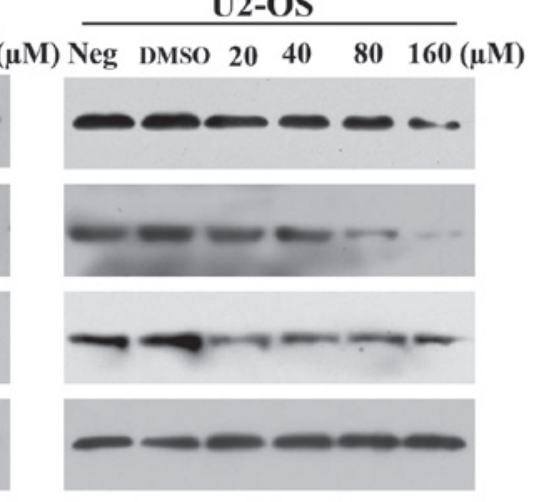

Figure 4. LY294002 inhibits the activity of the phosphatidylinositol 3-kinase/Akt/FASN signaling pathway in OS cells. (A) Representative images of the three experiments for each group. FASN mRNA expression was significantly downregulated with increasing concentrations of LY294002. (B) The results revealed that the p-Akt, Akt and FASN proteins in MG-63 and U2-OS cells treated with LY294002 were inhibited to varying degrees in a dose-dependent manner. FASN, fatty acid synthase; OS, osteosarcoma.

and FASN protein expression levels in cells treated with LY294002 were significantly lower compared with untreated cells (Fig. 4B). The present findings indicated that LY294002 downregulates the activation of the PI3K/Akt/FASN pathway.

\section{Discussion}

A significant number of studies on OS were published during the 1950s and 1960s, revealing poor 5-year survival rates of $<15 \%$ (17-19). Following the discovery of effective chemotherapy, the 5-year survival rates for patients treated with intensive multi-drug chemotherapy and aggressive local control have been reported to be $55-80 \%$ (20-22). However, chemotherapy fails to eliminate all OS cells due to intrinsic or acquired drug resistance, which is the most common cause of tumor recurrence and resultant poor clinical outcomes (23). Therefore, there is an urgent requirement to develop new drugs with improved chemotherapeutic effects for the management of OS.

LY294002, a selective inhibitor of PI3K, is the first artificially synthesized small molecular inhibitor of $\mathrm{PI} 3 \mathrm{~K} \alpha / \beta / \delta$, which acts on the adenosine triphosphate-binding site of the enzyme and is also more stable than wortmannin in solution. LY294002 inhibits cell proliferation and induces apoptosis 
by downregulating the activation of AKT/PKB. Semba et al demonstrated that LY294002 inhibits cell growth and induces apoptosis by decreasing the phosphorylation of Akt (Ser473) in colon cancer cell lines (24). Previous studies revealed that LY294002 inhibits cell growth and induces apoptosis in various types of cancer $(25,26)$. However, the mechanism of the anti-OS effect of LY294002 remains to be elucidated. In the present study, the results demonstrated that LY294002 induces MG-63 and U2-OS cell apoptosis and inhibits growth in a dose-dependent manner. Furthermore, the inhibitory effect of LY294002 on MG-63 and U2-OS cell migration and invasion was also revealed. The present findings suggest that LY294002 inhibits the malignant phenotype of OS cells in vitro and LY294002 may be an alternative treatment option for the management of OS.

The role of the PI3K/Akt signaling pathway in OS invasion and migration has been confirmed. Substantiated studies demonstrate that LY294002 inhibits malignant phenotypes of cells in various types of tumor via modulating the activity of the PI3K/Akt signaling pathway $(25,27)$. The molecular mechanisms associated with LY294002 inhibition of the malignant phenotype of OS cells were also analyzed. The current results demonstrated that the Akt and p-Akt protein in MG-63 and U2-OS cells was inhibited by LY294002 in a dose-dependent manner. It indicated that LY294002 may modulate the activation of the PI3K/Akt signaling pathway in OS cells.

FASN is an enzyme crucial for endogenous lipogenesis in mammals as it is responsible for catalyzing the synthesis of long-chain fatty acids. FASN is increased in a variety of human tumors and has been demonstrated to be associated with tumor cell growth, apoptosis and metastasis (28-31). It has been previously demonstrated that inhibition of FASN through use of pharmacological inhibitors or RNA interference significantly inhibits OS cell growth, migration and invasion in vitro and in vivo $(32,33)$. Previous studies have demonstrated that the activation of the PI3K/Akt pathway modulates FASN expression in tumor cells $(32,33)$. It is therefore hypothesized that the inhibition of the PI3K/Akt signaling pathway by LY294002 may modulate the expression of FASN, resulting in inhibition of the malignant phenotype in OS cells. To confirm these hypotheses, the inhibitory effect of LY294002 on the expression of FASN mRNA and protein was investigated by RT-qPCR and western blot analysis. The results revealed that the expression of FASN was inhibited by LY294002 in a dose-dependent manner.

Taken together, the present findings indicated LY294002 suppressed the malignant phenotype of OS cells by downregulating the PI3K/Akt/FASN signaling pathway in vitro. However, the tumor microenvironment is important in the malignant phenotype of tumor cells. Further experiments are required to elucidate whether LY294002 may be a new, alternative drug in vivo and whether targeting the PI3K/Akt/FASN pathway may be a potential treatment strategy for treating OS.

\section{Acknowledgements}

The present study was supported by grants from the National Natural Science Foundation of China (grant no. 81260400), the Natural Science Foundation of Jiangxi Province (grant no. 20114BAB205093) and the Jiangxi Provincial Department of Public Health (grant no. 20143052).

\section{References}

1. Mirabello L, Troisi RJ and Savage SA: Osteosarcoma incidence and survival rates from 1973 to 2004: data from the Surveillance, Epidemiology, and End Results Program. Cancer 115: 1531-1543, 2009.

2. Bacci G, Briccoli A, Rocca M, et al: Neoadjuvant chemotherapy for osteosarcoma of the extremities with metastases at presentation: recent experience at the Rizzoli Institute in 57 patients treated with cisplatin, doxorubicin, and a high dose of methotrexate and ifosfamide. Ann Oncol: 14: 1126-1134, 2003.

3. Ta HT, Dass CR, Choong PF and Dunstan DE: Osteosarcoma treatment: state of the art. Cancer metastasis Rev 28: 247-263, 2009.

4. Kager L, Zoubek A, Potschger U, et al: Primary metastatic osteosarcoma: presentation and outcome of patients treated on neoadjuvant Cooperative Osteosarcoma Study Group protocols. J Clin Oncol 21: 2011-2018, 2003.

5. Wang TF, Wang H, Peng AF, et al: Inhibition of fatty acid synthase suppresses U-2 OS cell invasion and migration via downregulating the activity of HER $2 / \mathrm{PI} 3 \mathrm{~K} / \mathrm{AKT}$ signaling pathway in vitro. Biochem Biophys Res Commun 440: 229-234, 2013.

6. Chen HJ, Lin CM, Lee CY, et al: Kaempferol suppresses cell metastasis via inhibition of the ERK-p38-JNK and AP-1 signaling pathways in U-2 OS human osteosarcoma cells. Oncol Rep 30: 925-932, 2013.

7. Zhao Z, Wu MS, Zou C, et al: Downregulation of MCT1 inhibits tumor growth, metastasis and enhances chemotherapeutic efficacy in osteosarcoma through regulation of the NF-kappaB pathway. Cancer Lett 342: 150-158, 2013.

8. Zhang S, Guo W, Ren TT and Lu XC, Tang GQ and Zhao FL: Arsenic trioxide inhibits Ewing's sarcoma cell invasiveness by targeting p38 (MAPK) and c-Jun N-terminal kinase. Anticancer Drugs 23: 108-118, 2012.

9. Liu R, Li X, Gao W, et al: Monoclonal antibody against cell surface GRP78 as a novel agent in suppressing PI3K/AKT signaling, tumor growth and metastasis. Clin Cancer Res 19: 6802-6811, 2013

10. Wang YH, Dong YY, Wang WM, et al: Vascular endothelial cells facilitated HCC invasion and metastasis through the Akt and NF-kappaB pathways induced by paracrine cytokines. J Exp Clin Cancer Res 32: 51, 2013.

11. Zito CR, Jilaveanu LB, Anagnostou V, et al: Multi-level targeting of the phosphatidylinositol-3-kinase pathway in non-small cell lung cancer cells. PLoS One 7: e31331, 2012.

12. Shukla S, Bhaskaran N, Babcook MA, Fu P, Maclennan GT and Gupta S: Apigenin inhibits prostate cancer progression in TRAMP mice via targeting PI3K/Akt/FoxO pathway. Carcinogenesis 35: 452-460, 2013.

13. Li J, Cao B, Zhou S, et al: Cyproheptadine-induced myeloma cell apoptosis is associated with inhibition of the PI3K/AKT signaling. Eur J Haematol 91: 514-521, 2013.

14. Qin H, Du X, Zhang Y and Wang R: Platycodin D, a triterpenoid saponin from Platycodon grandiflorum, induces G2/M arrest and apoptosis in human hepatoma HepG2 cells by modulating the PI3K/Akt pathway. Tumour Biol 35: 1267-1274, 2014.

15. Liu ZL, Mao JH, Peng AF, et al: Inhibition of fatty acid synthase suppresses osteosarcoma cell invasion and migration via downregulation of the PI3K/Akt signaling pathway in vitro. Mol Med Rep 7: 608-612, 2013.

16. Long XH, Zhang GM, Peng AF, Luo QF, Zhang L, Wen HC, et al: Lapatinib alters the malignant phenotype of osteosarcoma cells via downregulation of the activity of the HER2-PI3K/AKT-FASN axis in vitro. Oncol Rep Epub 2013/11/01, 2013.

17. McKenna RJ, Schwinn CP and Higinbotham NL: Osteogenic sarcoma in children. CA Cancer J Clin 16: 26-28, 1966.

18. Fraser GM and Aterman K: Osteogenic Sarcoma in Young Children: A Case Report with Review of Literature. Can Med Assoc J 87: 74-78, 1962.

19. Pearson D and Steward JK: Malignant disease in juveniles. Proc R Soc Med 62: 685-688, 1969.

20. Iwamoto Y, Tanaka K, Isu K, et al: Multiinstitutional phase II study of neoadjuvant chemotherapy for osteosarcoma (NECO study) in Japan: NECO-93J and NECO-95J. J Orthop Sci 14: 397-404, 2009. 
21. Meyers PA, Schwartz CL, Krailo M, et al: Osteosarcoma: a randomized, prospective trial of the addition of ifosfamide and/or muramyl tripeptide to cisplatin, doxorubicin, and high-dose methotrexate. J Clin Oncol 23: 2004-2011, 2005.

22. Jawad MU, Cheung MC, Clarke J, Koniaris LG and Scully SP: Osteosarcoma: improvement in survival limited to high-grade patients only. J Cancer Res Clin Oncol 137: 597-607, 2011.

23. Ferrari S, Smeland S, Mercuri M, et al: Neoadjuvant chemotherapy with high-dose Ifosfamide, high-dose methotrexate, cisplatin, and doxorubicin for patients with localized osteosarcoma of the extremity: a joint study by the Italian and Scandinavian Sarcoma Groups. J Clin Oncol 23: 8845-8852, 2005.

24. Semba S, Itoh N, Ito M, Harada M and Yamakawa M: The in vitro and in vivo effects of 2-(4-morpholinyl)-8-phenyl-chromone (LY294002), a specific inhibitor of phosphatidylinositol 3'-kinase, in human colon cancer cells. Clin Cancer Res 8: 1957-1963, 2002.

25. Chen Z, Yang L, Liu Y, et al: LY294002 and Rapamycin promote coxsackievirus-induced cytopathic effect and apoptosis via inhibition of PI3K/Akt/mTOR signaling pathway. Mol Cell Biochem 385: 169-177, 2013.

26. Hu L, Zaloudek C, Mills GB, Gray J and Jaffe RB: In vivo and in vitro ovarian carcinoma growth inhibition by a phosphatidylinositol 3-kinase inhibitor (LY294002). Clin Cancer Res 6: 880-886, 2000.

27. Wempe SL, Gamarra-Luques CD and Telleria CM: Synergistic lethality of mifepristone and LY294002 in ovarian cancer cells. Cancer Growth Metastasis 6: 1-13, 2013.
28. Liu ZL, Wang G, Peng AF, Luo QF, Zhou Y and Huang SH: Fatty acid synthase expression in osteosarcoma and its correlation with pulmonary metastasis. Oncol Lett 4: 878-882, 2012.

29. Porta R, Blancafort A, Casoliva G, et al: Fatty acid synthase expression is strongly related to menopause in early-stage breast cancer patients. Menopause 21: 188-191, 2013.

30. Tao BB, He H, Shi XH, et al: Up-regulation of USP2a and FASN in gliomas correlates strongly with glioma grade. J Clin Neurosci 20: 717-720, 2013.

31. Rahman MT, Nakayama K, Ishikawa M, et al: Fatty acid synthase is a potential therapeutic target in estrogen receptor-/progesterone receptor-positive endometrioid endometrial cancer. Oncology 84: 166-173, 2013.

32. Liu ZL, Wang G, Shu Y, Zou PA, Zhou Y and Yin QS: Enhanced antitumor activity of epirubicin combined with cerulenin in osteosarcoma. Mol Med Rep 5: 326-330, 2012.

33. Liu ZL, Zhou Y, Luo QF, et al: Inhibition of fatty acid synthase supresses osteosarcoma cell invasion and migration. Indian J Pathol Microbiol 55: 163-169, 2012.

34. Yeh CW, Chen WJ, Chiang CT, Lin-Shiau SY and Lin JK: Suppression of fatty acid synthase in MCF-7 breast cancer cells by tea and tea polyphenols: a possible mechanism for their hypolipidemic effects. Pharmacogenomics J 3: 267-276, 2003.

35. Van de Sande T, De Schrijver E, Heyns W, Verhoeven G and Swinnen JV: Role of the phosphatidylinositol 3'-kinase/PTEN/Akt kinase pathway in the overexpression of fatty acid synthase in LNCaP prostate cancer cells. Cancer Res 62: 642-646, 2002. 